\title{
Radiolabeled (4-Fluoro-3-Iodobenzyl)Guanidine Improves Imaging and Targeted Radionuclide Therapy of Norepinephrine Transporter-Expressing Tumors
}

\author{
Aiko Yamaguchi ${ }^{1}$, Hirofumi Hanaoka ${ }^{1}$, Tetsuya Higuchi ${ }^{2}$, and Yoshito Tsushima ${ }^{2}$ \\ ${ }^{1}$ Department of Bioimaging Information Analysis, Gunma University Graduate School of Medicine, Maebashi, Japan; and \\ ${ }^{2}$ Department of Diagnostic Radiology and Nuclear Medicine, Gunma University Graduate School of Medicine, Maebashi, Japan
}

\begin{abstract}
${ }^{18} \mathrm{~F}$ - or ${ }^{131} \mathrm{I}$-labeled (4-fluoro-3-iodobenzyl)guanidine (FIBG) has been a promising yet unattainable derivative of radioiodine-labeled meta-iodobenzylguanidine (MIBG), because of the complex radiofluorination method. In this study, we proposed a 2-step radiosynthetic method to obtain ${ }^{18} \mathrm{~F}-\mathrm{FIBG}$ and evaluated the diagnostic and therapeutic potential of ${ }^{18} \mathrm{~F}-\mathrm{FIBG}$ and ${ }^{131} \mathrm{I}-\mathrm{FIBG}$ in a pheochromocytoma model (PC-12). Methods: ${ }^{18} \mathrm{~F}-\mathrm{FIBG}$ was prepared from a (mesityl)(aryl)iodonium salt precursor in the presence of a copper catalyst. Biodistribution studies, PET imaging, and a therapeutic study were performed on the PC-12 xenograft mice with either ${ }^{18} \mathrm{~F}$ - or ${ }^{131} \mathrm{I}$-FIBG. The association between the therapeutic effect and the tumor uptake of pretherapy ${ }^{18} \mathrm{~F}$-FIBG PET was also evaluated. Results: The coppermediated radiofluorination method readily yielded ${ }^{18} \mathrm{~F}-\mathrm{FIBG}$, as well as its regioisomer, ${ }^{18} \mathrm{~F}-\mathrm{IFBG}$. The isolated ${ }^{18} \mathrm{~F}-\mathrm{FIBG}$ showed a higher accumulation in the PC-12 xenograft tumor than in any other tissue. The high tumor uptake of ${ }^{18} \mathrm{~F}-\mathrm{FIBG}$ allowed clear tumor visualization in the PET images as early as $1 \mathrm{~h}$ after injection, with an excellent tumorto-background ratio. A biodistribution study with ${ }^{131}$ I-FIBG revealed its higher and prolonged retention in the tumor in comparison with 125I-MIBG. As a result, a therapeutic dose of ${ }^{131}$ I-FIBG delayed tumor growth significantly more than did ${ }^{131}$ I-MIBG. The tumor uptake of ${ }^{18} \mathrm{~F}$-FIBG was proportional to the therapeutic effect of ${ }^{131}$ I-FIBG. Conclusion: These results suggest the potential usefulness of FIBG as a diagnostic and therapeutic agent for the management of norepinephrine transporter (NET)-expressing tumors.
\end{abstract}

Key Words: pheochromocytoma; neuroblastoma; MIBG analog; PET; targeted radionuclide therapy

J Nucl Med 2018; 59:815-821

DOI: 10.2967/jnumed.117.201525

\section{$\mathbf{R}$} adioiodine-labeled meta-iodobenzylguanidine (MIBG) plays a role in the diagnosis and treatment of neuroblastomas and malignant pheochromocytomas (1-3). ${ }^{123} \mathrm{I}-\mathrm{MIBG}$ is preferred for diagnostic imaging, whereas ${ }^{131} \mathrm{I}-\mathrm{MIBG}$ therapy has produced a noticeable response rate: most patients with malignant pheochromocytomas achieve disease stabilization, and approximately $30 \%$

Received Aug. 31, 2017; revision accepted Nov. 16, 2017.

For correspondence or reprints contact: Hirofumi Hanaoka, Gunma University Graduate School of Medicine, 3-39-22 Showa, Maebashi, Gunma 371-8511 Japan.

E-mail: hanaokah@gunma-u.ac.jp

Published online Dec. 7, 2017.

COPYRIGHT (C 2018 by the Society of Nuclear Medicine and Molecular Imaging. of relapsed or refractory patients with neuroblastomas achieve a complete or partial response to therapy. Despite this clinical usefulness, both of these radioiodine-labeled MIBGs have their limitations $(1,2,4)$, and attempts have therefore been made to develop alternative MIBG derivatives (5-8).

Among the MIBG derivatives developed thus far, a promising pair of candidates is the combination of ${ }^{18} \mathrm{~F}$ - and ${ }^{131} \mathrm{I}$-labeled (4-fluoro-3-iodobenzyl)guanidine (FIBG) (Fig. 1A) (8). Although both MIBGs and FIBGs can offer integrated diagnosis and therapy by the structurally identical agents, FIBGs may provide additional value because they allow radiolabeling with ${ }^{18} \mathrm{~F}$, the most frequently used positron-emitter isotope. The diagnostic superiority of a PET probe over a single-photon probe for image-guided therapy is exemplified by the success of radiolabeled peptides targeting somatostatin receptors (9). The MIBG derivatives evaluated in this study, ${ }^{18} \mathrm{~F}$ - and ${ }^{131} \mathrm{I}-\mathrm{FIBG}$, showed basic properties comparable or superior to MIBG in cellular and normal-mouse studies $(10,11)$. However, likely because of the complex radiosynthesis of ${ }^{18} \mathrm{~F}$-FIBG $(8)$, further properties, such as tumor uptake and the therapeutic effectiveness of ${ }^{131}$ I-FIBG, remain unknown.

Owing to recent developments in transition-metal-mediated radiofluorination methods for arenes, simple preparations of various ${ }^{18} \mathrm{~F}$-fluoroarenes have become possible, including probes that were previously difficult to develop (12). Given the applicability of these techniques to the radiosynthesis of another MIBG analog, meta- ${ }^{18} \mathrm{~F}$-fluorobenzylguanidine $(13-15)$, we hypothesized that the synthesis of ${ }^{18}$ F-FIBG could be simplified by adapting one of these methods.

In this study, we developed a 2 -step ${ }^{18} \mathrm{~F}$-FIBG preparation method using the copper-mediated fluorination of mesityl(aryl) iodonium salt, a technique developed by Ichiishi et al. (16). The usefulness of ${ }^{18} \mathrm{~F}-\mathrm{FIBG}$ for the detection and ${ }^{131} \mathrm{I}-\mathrm{FIBG}$ for the treatment of norepinephrine transporter (NET)-expressing tumors was evaluated using pheochromocytoma xenograft mice.

\section{MATERIALS AND METHODS}

Unless otherwise stated, reagents and solvents were commercially available and used without further purification. Reagents were purchased from Sigma-Aldrich, TCI, or Wako. No-carrier-added radioiodinated MIBG derivatives were prepared as previously described (17).

\section{Synthesis of Iodonium Salt Precursors (Compounds 6a and 6b)}

The mesityl-iodonium salt precursors were prepared according to published methods $(15,16)$ with slight modifications (Fig. 1B). A cold fluorination reaction was performed as previously described 


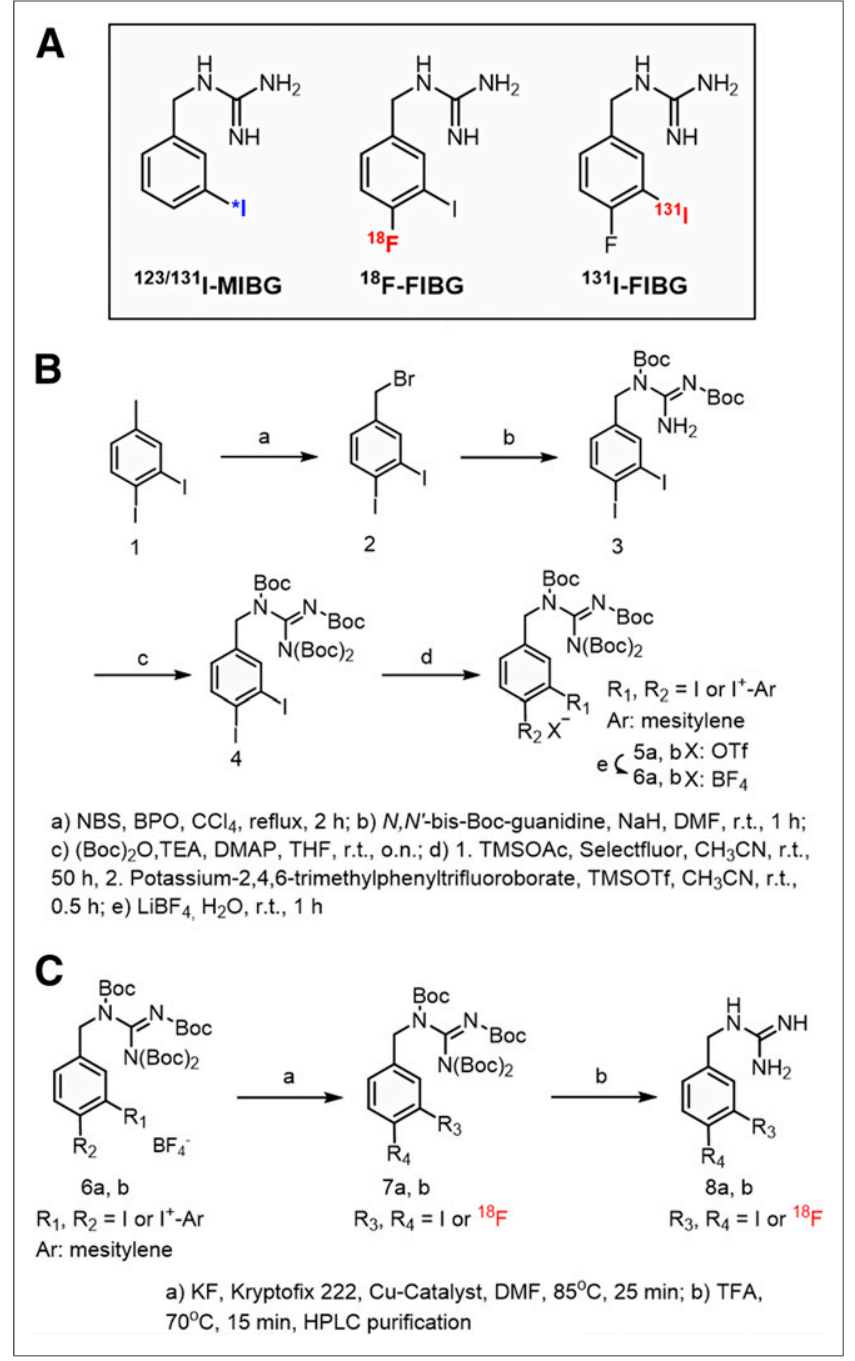

FIGURE 1. (A) Chemical structures of radiolabeled MIBG and FIBG. (B) Synthesis of $\mathbf{6 a}$ and $\mathbf{6 b}$. (C) Radiosynthesis of $\mathbf{8 a}$ and $\mathbf{8 b}$. Boc = tertbutoxycarbonyl; (Boc)2O = di-tert-butyl dicarbonate; BPO = benzoyl peroxide; DAMP $=4$-(dimethylamino)pyridine; DMF $=N, N$-dimethylformamide; NBS $=\mathrm{N}$-bromosuccinimide; TEA $=$ triethylamine; TFA $=$ trifluoroacetic acid; THF = tetrahydrofuran; TMSOAc = trimethylsilyl acetate; TMSOTf $=$ trimethylsilyl triflate.

(Supplemental Fig. 1A; supplemental materials are available at http:// jnm.snmjournals.org) (16).

\section{Synthesis of ${ }^{18} \mathrm{~F}$-FIBG and ${ }^{18} \mathrm{~F}$-IFBG (Compounds $8 \mathrm{a}$ and $8 \mathrm{~b}$ )}

${ }^{18} \mathrm{~F}$-fluoride was produced via the ${ }^{18} \mathrm{O}(\mathrm{p}, \mathrm{n}){ }^{18} \mathrm{~F}$ nuclear reaction using a cyclotron at Gunma University Hospital. The ${ }^{18} \mathrm{~F}$ labeling of the intermediate compounds (7a and 7b) was performed as previously described (16) with subtle modifications (Fig. 1C). A 2-mL volume of water and a grain of Chelex 100 chelating resin (Bio-Rad) were then added to the reaction vial to trap the copper ion. The mixture was diluted with an additional 10 $\mathrm{mL}$ of water and subjected onto a C18 Sep-Pak cartridge (Waters Corp.) via a filtration unit. Unreacted ${ }^{18} \mathrm{~F}$ was washed with an additional $10 \mathrm{~mL}$ of water, and the column was dried with $\mathrm{N}_{2}$. The mixture (7a and $7 \mathbf{b}$ ) was eluted with $1.5 \mathrm{~mL}$ of $\mathrm{CH}_{3} \mathrm{CN}$ into a glass tube. The elution was evaporated with a stream of $\mathrm{N}_{2}$ at $60^{\circ} \mathrm{C}$, and then the deprotection reaction was performed with trifluoroacetic acid at $75^{\circ} \mathrm{C}$. After $15 \mathrm{~min}$, the solvent was evaporated with a stream of $\mathrm{N}_{2}$. The resulting regioisomer mixture of
${ }^{18} \mathrm{~F}-\mathrm{FIBG}$ and ${ }^{18} \mathrm{~F}-$ IFBG (8a and $\mathbf{8 b}$ ) was diluted with approximately $1 \mathrm{~mL}$ of $25 / 75 \mathrm{MeOH} / \mathrm{H}_{2} \mathrm{O}$, and the regioisomers were separated using semipreparative high-performance liquid chromatography (HPLC). The radiochemical yield (RCY) of the intermediate compound (7a and $\mathbf{7 b}$ ) was determined by dividing the integrated area under the objective compound spot by the total integrated area of the thin-layer chromatography plate (Supplemental Fig. 2).

\section{Cellular Studies for MIBG Derivatives}

The rat pheochromocytoma cell line PC-12 was obtained from the American Type Culture Collection. The cellular uptake studies for ${ }^{18} \mathrm{~F}-$ FIBG and ${ }^{18} \mathrm{~F}$-IFBG were performed as previously described (10). The cellular uptake and release profiles for ${ }^{131}$ I-MIBG and ${ }^{131}$ I-FIBG in a 3-dimensional cell culture model, and the cellular therapeutic study for ${ }^{131}$ I-FIBG and ${ }^{131}$ I-MIBG, were performed as previously described (11) with subtle modifications. For a 3-dimensional culture model, the PC-12 cells were seeded into 96-well Nunclon Sphera microplates (Thermo Fisher Scientific, $1.0 \times 10^{5}$ cells per well) $24 \mathrm{~h}$ before the experiments.

\section{Preparation of PC-12-Bearing Mice}

All experimental protocols were approved by the Laboratory Animal Care and Use Committee of Gunma University. Five- to 7-wk-old female BALB/c nu/nu mice (Japan SLC) were inoculated with PC-12 cells $\left(5 \times 10^{6}\right.$ cells per mouse) in the flank. Approximately 3 wk after inoculation, the experiments described below were performed.

\section{Tissue Distribution in Mice}

Two studies were conducted on PC-12-bearing mice. First, a biodistribution study was performed in a paired-label format by coinjecting the mice with $50 \mathrm{kBq}$ of ${ }^{18} \mathrm{~F}$-labeled and $5 \mathrm{kBq}$ of ${ }^{125}$ I-labeled MIBG derivatives $\left({ }^{18} \mathrm{~F}\right.$-FIBG $+{ }^{125} \mathrm{I}$-FIBG, ${ }^{18} \mathrm{~F}$-IFBG $+{ }^{125} \mathrm{I}$-IFBG, or the regioisomer mixture $+{ }^{125}$ I-MIBG; 4-5 mice per group) at $1 \mathrm{~h}$ after injection. Next, a biodistribution study was also performed in a paired-label format by coinjecting the mice with $5 \mathrm{kBq}$ each of ${ }^{125}$ I-MIBG and ${ }^{131}$ I-FIBG at 1 , $3,6,24$, and $48 \mathrm{~h}$ (4-5 mice per group), as well as at $5 \mathrm{~d}$, after injection ( 3 mice per group). In both studies, the mice were humanely killed by decapitation. The tissues of interest were isolated and weighed. A $\gamma$-counter (ARC7001; Hitachi Aloka Medical) was used to determine ${ }^{18} \mathrm{~F},{ }^{125} \mathrm{I}$, or ${ }^{131}$ I activity. Another biodistribution study was performed on normal mice with the injection of $50 \mathrm{kBq}$ each of ${ }^{125}$ I-FIBG or ${ }^{125} \mathrm{I}-\mathrm{MIBG}$, and the absorbed radiation dose in humans was estimated using OLINDA/EXM, version 1.1 (Vanderbilt University).

\section{PET Imaging and Image Analysis}

The PC-12-bearing mice were intravenously administered 1-2 MBq of ${ }^{18} \mathrm{~F}-\mathrm{FIBG},{ }^{18} \mathrm{~F}-\mathrm{IFBG}$, or the regioisomer mixture. The mice were anesthetized using isoflurane inhalation, and PET scans were performed 1 and $2 \mathrm{~h}$ after administration of the MIBG derivative using a small-animal PET scanner (Inveon; Siemens) with 10-min emission scanning. For ${ }^{18} \mathrm{~F}-\mathrm{FDG}$ PET imaging, the PC-12-bearing mice were intravenously administered $5 \mathrm{MBq}$ of ${ }^{18} \mathrm{~F}-\mathrm{FDG}$ after a 12 -h fast, on the next day of ${ }^{18} \mathrm{~F}$-FIBG PET imaging. PET imaging was performed as described above. Semiquantitative analysis was performed for each identified tumor using AMIDE software (Stanford University). Volumes of interest were drawn manually to trace the contours of the tumor without correcting for partial-volume effects. The background region of interest was placed in the muscles surrounding the shoulder. The mean tumor uptake was divided by the mean background uptake to calculate the tumor-to-background ratio. $\mathrm{SUV}_{\max }$ was determined using an Inveon Research Workplace workstation (Siemens).

\section{Therapeutic Study In Vivo}

When the tumors were fully established $\left(0.16 \pm 0.08 \mathrm{~cm}^{3}\right)$, the animals were randomly assigned to groups. There was no significant difference in average tumor size between groups. The mice were intravenously administered ${ }^{131} \mathrm{I}$-MIBG $(10 \mathrm{MBq})$ or ${ }^{131} \mathrm{I}$-FIBG $(1,3,5$, 
or $10 \mathrm{MBq}$ ). Saline-injected mice were used as controls. At least 5 mice were allotted to each group, with the exception of the ${ }^{131}$ I-FIBG 1-MBq group, which consisted of only 3 mice. The weight and tumor diameters of the mice were measured regularly. The tumor volumes were determined using the following formula: (length) $\times(\text { width })^{2} \times 0.5$. Each tumor volume was divided by the initial tumor volume to determine the relative tumor volume. To evaluate the relationship between ${ }^{18} \mathrm{~F}-\mathrm{FIBG}$ uptake and the therapeutic effect of ${ }^{131}$ I-FIBG, $5 \mathrm{MBq}$ of ${ }^{131} \mathrm{I}$-FIBG were injected into each animal on the next day of ${ }^{18} \mathrm{~F}-\mathrm{FDG}$ PET imaging ( $2 \mathrm{~d}$ after the ${ }^{18} \mathrm{~F}$-FIBG PET imaging), and the animals were monitored as described above.

\section{Statistical Analysis}

GraphPad Prism was used for the statistical analyses. Data are presented as mean $\pm \mathrm{SD}$. Statistical significance was determined on the basis of the Student $t$ test (a 2-sample test or a paired test) or ANOVA with the Tukey post hoc test when appropriate. Simple correlation between variables was analyzed using the Pearson correlation coefficient. Values of $P$ that were less than 0.05 were considered statistically significant.

\section{RESULTS}

\section{Synthesis of Iodonium Salt Precursors (Compounds 6a and $6 \mathrm{~b}$ )}

The direct 1-pot oxidation/iodine arylation of 4 with mesytil$\mathrm{BF}_{3} \mathrm{~K}$ yielded a mixture of $p$ - and $m$-substituted asymmetric diaryliodonium triflates $(\mathbf{5 a}$ and $\mathbf{5 b}$ ), which were difficult to separate by silica gel chromatography. Because attempts to produce isomerically pure 5a failed, a regioisomer mixture was used in the following experiments. An anion metathesis reaction with saturated aqueous $\mathrm{LiBF}_{4}$ produced a free-flowing powder of mesityl(aryl)iodonium tetrafluoroborate as a regioisomer mixture $(\mathbf{6 a}$ and $\mathbf{6 b})$, with an overall yield of $12.7 \%$. The subsequent ${ }^{19} \mathrm{~F}$ nuclear magnetic resonance analysis of the cold fluorination reaction product revealed that the mixture readily gave a 3:2 ratio of tetra-Boc-protected FIBG and tetra-Boc-protected IFBG (Supplemental Fig. 1B).

\section{Synthesis of ${ }^{18} \mathrm{~F}$-FIBG and ${ }^{18} \mathrm{~F}$-IFBG (Compounds $8 \mathrm{a}$ and $8 \mathrm{~b}$ )}

We then attempted ${ }^{18} \mathrm{~F}$ labeling of 6 (step a in Fig. 1C). Although the procedure in the literature (16) provided the intermediate compounds (7a and $\mathbf{7 b}$ ), the RCY and reproducibility of the reaction were low $(13.6 \% \pm 10 \%, n=4)$. By increasing the amount of $\mathrm{Cu}(\mathrm{MeCN})_{4} \mathrm{OTf}$ to 5 equivalents of the iodonium salt 6, we obtained the highest RCY $(63.9 \%)$, yet with unsatisfying reproducibility $(18.2 \% \pm 19.7 \%, n=14)$. In contrast, when we

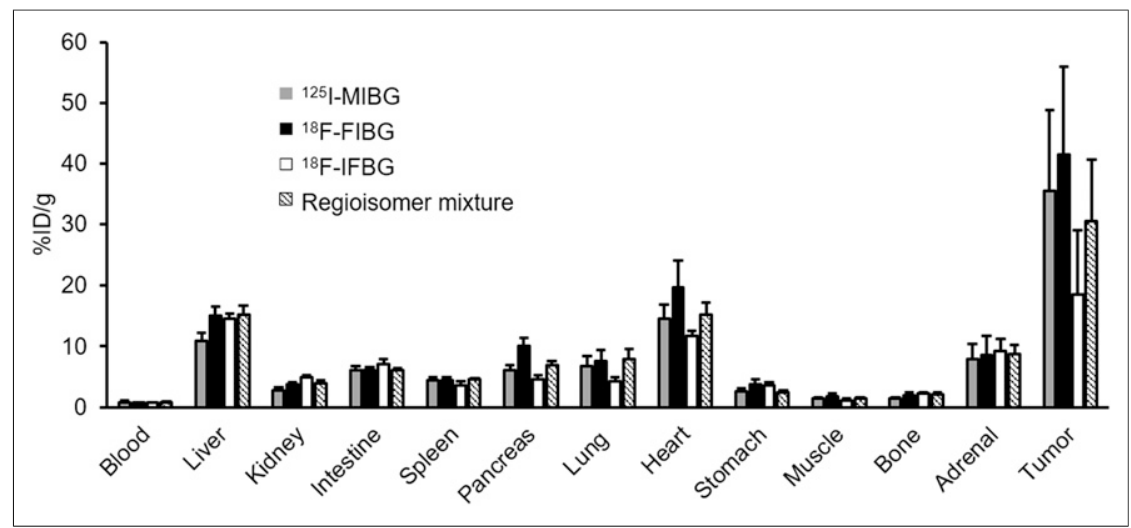

FIGURE 2. Biodistribution profiles of ${ }^{18} \mathrm{~F}$ - and ${ }^{125} \mathrm{I}-\mathrm{MIBG}$ derivatives in PC-12-bearing mice at $1 \mathrm{~h}$ after injection (mean \pm SD, 4-5 per group). used an alternative copper catalyst $\left(\mathrm{Cu}(\mathrm{OTf})_{2}\right)$, a fair RCY was obtained, with moderate reproducibility $(19.7 \% \pm 8.0 \%, n=13)$. In the following deprotection reaction (Fig. 1C), we first tried to replicate the previously developed procedure by directly adding $6 \mathrm{~N} \mathrm{HCl}$ to the reaction mixture and incubating it at $120^{\circ} \mathrm{C}(15)$. However, a large degree of defluorination and the formation of multiple by-products decreased the recovery yield of $\mathbf{8}$ from $\mathbf{7}$ to less than $50 \%$. By removing copper and unreacted ${ }^{18} \mathrm{~F}^{-}$, and by reducing the temperature to $70^{\circ} \mathrm{C},{ }^{18} \mathrm{~F}-\mathrm{FIBG}$ and ${ }^{18} \mathrm{~F}$-IFBG $(\mathbf{8 a}$ and $\mathbf{8 b}$ ) could be obtained in almost quantitative yield from $7 \mathbf{a}$ and 7b. The regioisomer separation was achieved using semipreparative HPLC with high radiochemical purity ( $>99 \%$, Supplemental Fig. 3). The products $\mathbf{8 a}$ and $\mathbf{8 b}$ were obtained with an overall RCY of $5.06 \% \pm 1.73 \%$ and $2.75 \% \pm 0.95 \%$, respectively (decaycorrected, $n=4)$.

\section{In Vitro Profiles of Radiolabeled MIBG Derivatives in PC-12 Cells}

The accumulation of ${ }^{18} \mathrm{~F}-\mathrm{FIBG}$ in pheochromocytoma cells was approximately 1.5 -fold higher than that of ${ }^{125}$ I-MIBG (Supplemental Fig. 4A), whereas the accumulation of ${ }^{18} \mathrm{~F}$-IFBG was approximately 0.75 -fold lower than that of ${ }^{125} \mathrm{I}-\mathrm{MIBG}$. The uptake of all compounds was decreased by NET inhibitors to comparable levels (Supplemental Fig. 4B). The uptake level of ${ }^{125}$ I-FIBG was also higher than that of ${ }^{131}$ I-MIBG in the 3-dimensional cell culture model, and a comparable release profile was found between ${ }^{131} \mathrm{I}$-FIBG and ${ }^{131} \mathrm{I}-\mathrm{MIBG}$ (Supplemental Figs. 5A and 5B, respectively). As shown in Supplemental Figure 5C, ${ }^{131} \mathrm{I}-\mathrm{FIBG}$ exhibited greater cytotoxic effects at doses higher than $1 \mathrm{MBq} / \mathrm{mL}$.

\section{Biodistribution of ${ }^{18} \mathrm{~F}$-FIBG and ${ }^{18} \mathrm{~F}$-IFBG in Mice}

We next evaluated the biodistribution profiles of ${ }^{18} \mathrm{~F}-\mathrm{FIBG},{ }^{18} \mathrm{~F}-$ IFBG, and the regioisomer mixture in comparison with ${ }^{125} \mathrm{I}-\mathrm{MIBG}$ (Fig. 2) in PC-12-bearing mice $1 \mathrm{~h}$ after injection. All fluorinated compounds showed the highest uptake within the tumor, similar to the distribution pattern of MIBG. Tumor uptake of ${ }^{18} \mathrm{~F}$-FIBG was slightly higher than that of ${ }^{125} \mathrm{I}-\mathrm{MIBG}$, although the difference was not statistically significant $(41.5 \pm 14.5$ vs. $35.4 \pm 13.3$ percentage injected dose [\% ID]/g, respectively). In contrast, tumor uptake of ${ }^{18} \mathrm{~F}-$ IFBG was approximately half that of ${ }^{18} \mathrm{~F}-\mathrm{FIBG}(18.6 \pm 10.4 \%$ $\mathrm{ID} / \mathrm{g}, P<0.05)$. There was no significant difference between the tumor uptake of the regioisomer mixture and ${ }^{125}$ I-MIBG. Comparable biodistribution profiles were found between ${ }^{18} \mathrm{~F}-\mathrm{FIBG}$ and ${ }^{125} \mathrm{I}$-FIBG and between ${ }^{18} \mathrm{~F}$-IFBG and ${ }^{125} \mathrm{I}$-IFBG (Supplemental Fig. 6). The biodistribution profiles of ${ }^{125} \mathrm{I}-$ FIBG and ${ }^{125}$ I-MIBG up to $7 \mathrm{~d}$ are shown in Supplemental Table 1. The calculated absorbed dose of the red marrow, the critical organ for ${ }^{131} \mathrm{I}-\mathrm{MIBG}$, was 8.70 and 5.03 $\mu \mathrm{Sv} / \mathrm{MBq}$ for ${ }^{131} \mathrm{I}-\mathrm{FIBG}$ and ${ }^{131} \mathrm{I}-\mathrm{MIBG}$, respectively. The effective dose equivalent was 125 and $80.9 \mu \mathrm{Sv} / \mathrm{MBq}$ for ${ }^{131} \mathrm{I}$-FIBG and ${ }^{131} \mathrm{I}-\mathrm{MIBG}$, respectively.

\section{PET Imaging}

To evaluate the tumor detectability of ${ }^{18} \mathrm{~F}$ FIBG and ${ }^{18} \mathrm{~F}$-IFBG, PET imaging was performed on PC-12-bearing mice (Fig. 3). As early as $1 \mathrm{~h}$ after injection, ${ }^{18} \mathrm{~F}-\mathrm{FIBG},{ }^{18} \mathrm{~F}$ IFBG, and the regioisomer mixture clearly depicted even small tumors $(4 \mathrm{~mm})$. The tumor-to-background ratios of ${ }^{18} \mathrm{~F}-\mathrm{FIBG}$, 


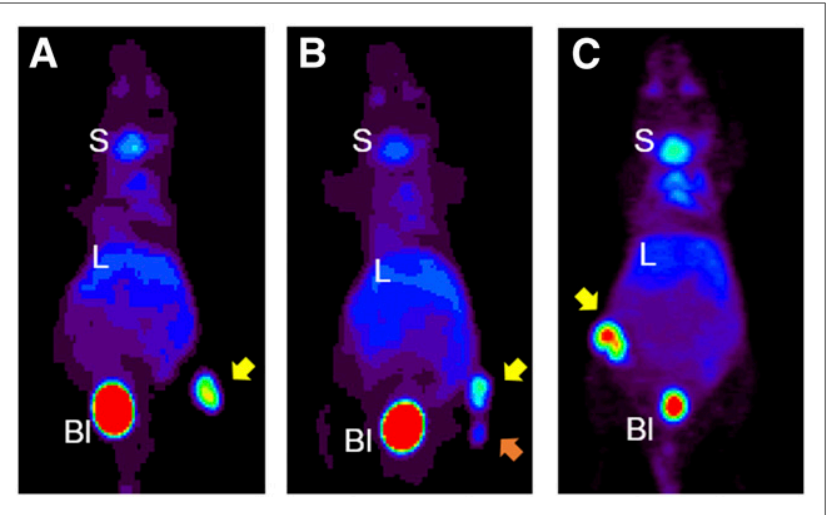

FIGURE 3. Representative PET images with ${ }^{18} \mathrm{~F}-\mathrm{FIBG}(\mathrm{A}),{ }^{18} \mathrm{~F}-\mathrm{IFBG}$ $(B)$, and regioisomer mixture $(C)$ in $\mathrm{PC}-12-$ bearing mice at $1 \mathrm{~h}$ after injection. Yellow arrows indicate tumor locations, and orange arrow indicates small tumor $(4 \mathrm{~mm}) . \mathrm{BI}=$ bladder; $\mathrm{L}=$ liver; $\mathrm{S}=$ salivary gland.

${ }^{18} \mathrm{~F}-\mathrm{IFBG}$, and the regioisomer mixture were $9.92 \pm 3.43(n=$ $12), 6.95 \pm 1.42(n=6)$, and $18.4(n=1)$, respectively. In the 2-h image, ${ }^{18} \mathrm{~F}$-FIBG showed improved tumor-to-background ratios (13.3 \pm 4.33, $n=12, P<0.05$; Supplemental Fig. 7).

\section{Tumor Retention of ${ }^{125}$ I-FIBG and ${ }^{131}$ I-MIBG in} PC-12-Bearing Mice up to 5 Days

The biodistribution profiles of ${ }^{125}$ I-FIBG and ${ }^{131}$ I-MIBG in PC12-bearing mice up to $5 \mathrm{~d}$ after injection are shown in Supplemental Table 2. Overall, ${ }^{125}$ I-FIBG showed a similar biodistribution profile to ${ }^{131} \mathrm{I}-\mathrm{MIBG}$, although the initial uptake was higher than for ${ }^{131} \mathrm{I}-$ MIBG in almost all organs. A relatively fast clearance of ${ }^{125}$ I-FIBG from nontarget organs (low NET-expressing) was noted. In contrast, in NET-positive tissues such as the heart, adrenal glands, and tumor, ${ }^{125} \mathrm{I}-\mathrm{FIBG}$ retention was longer and higher than ${ }^{131} \mathrm{I}-\mathrm{MIBG}$ retention for up to $5 \mathrm{~d}$. In particular, ${ }^{125}$ I-FIBG accumulation in the tumor was higher than in any other tissue during the entire observation period. The uptake peaked $24 \mathrm{~h}$ after injection $(83.9 \pm 7.37 \% \mathrm{ID} / \mathrm{g})$, with $18.4 \pm 3.78 \% \mathrm{ID} / \mathrm{g}$ remaining $5 \mathrm{~d}$ later. In contrast, the uptake of ${ }^{131}$ I-MIBG peaked 3-6 h after injection, with little remaining $5 \mathrm{~d}$ later (Fig. 4).

\section{Tumor Growth Inhibition Effect of ${ }^{131}$ I-FIBG}

The growth curves of PC-12 xenografts after administration of ${ }^{131}$ I-FIBG or ${ }^{131}$ I-MIBG are shown in Figure 5A. At doses higher than $3 \mathrm{MBq}$, a single treatment with ${ }^{131}$ I-FIBG suppressed the tumor growth rate as compared with the saline-injected control group $(P<0.0001)$. Injections of $10 \mathrm{MBq}$ of ${ }^{131} \mathrm{I}-\mathrm{MIBG}$ resulted in a 2.5 -fold increase in tumor volume $\left(\mathrm{T}_{2.5}\right) 22.6 \pm 2.88 \mathrm{~d}$ after injection (Supplemental Table 3). The same dose of ${ }^{131}$ I-FIBG delayed $\mathrm{T}_{2.5}$ until a later time $(31.7 \pm 2.88 \mathrm{~d}, P=0.033)$. Even at lower doses ( 3 and $5 \mathrm{MBq}$ ), $\mathrm{T}_{2.5}$ was later after administration for ${ }^{131}$ I-FIBG than for ${ }^{131} \mathrm{I}$-MIBG (10 MBq), although this difference was not statically significant. No significant difference was observed in the body weight change (Fig. 5B).

\section{Relationship Between ${ }^{18}$ F-FIBG Uptake and ${ }^{18}$ F-FDG Uptake and Between ${ }^{18} \mathrm{~F}$-FIBG Uptake and ${ }^{131}$ I-FIBG Growth Inhibition}

Reflecting the fact that xenograft tumors derived from PC-12 show various differentiation states and various uptake levels of MIBG derivatives (18), the PC-12 tumors showed various $\mathrm{SUV}_{\max }$ levels in the pretreatment ${ }^{18} \mathrm{~F}$-FIBG PET images. In the linear regression analysis of pretreatment PET imaging, ${ }^{18} \mathrm{~F}-\mathrm{FIBG}$ uptake and ${ }^{18} \mathrm{~F}$-FDG uptake showed a strong inverse correlation in the PC12 xenograft tumors $(n=9$, Pearson correlation $=-0.88, P<$ 0.05 ; Fig. 6A). Uptake of ${ }^{18} \mathrm{~F}-\mathrm{FIBG}$ also showed a strong inverse correlation with the relative volume of the PC-12 xenograft $33 \mathrm{~d}$ after treatment with ${ }^{131} \mathrm{I}$-FIBG $(n=9$, Pearson correlation $=-0.76$, $P<0.05$; Fig. 6B). Figures $6 \mathrm{C}$ and $6 \mathrm{D}$ show representative PET images with high ${ }^{18} \mathrm{~F}$-FIBG uptake and low ${ }^{18} \mathrm{~F}$-FDG uptake and with low ${ }^{18} \mathrm{~F}$-FIBG uptake and high ${ }^{18} \mathrm{~F}$-FDG uptake, respectively. In addition to the contrasting levels in these tumors, ${ }^{18} \mathrm{~F}$-FIBG and ${ }^{18} \mathrm{~F}-\mathrm{FDG}$ showed visibly different intratumoral distributions. Change in tumor size between the 2 scans was negligible.

\section{DISCUSSION}

This study established a 2-step radiofluorination method to produce ${ }^{18} \mathrm{~F}$-FIBG, a promising diagnostic agent for NET-expressing tumors. Despite the structural similarities, the additional iodine in ${ }^{18} \mathrm{~F}$-FIBG made it challenging to apply the radiofluorination methods used for the production of meta- ${ }^{18} \mathrm{~F}$-fluorobenzylguanidine (13-15). Indeed, the additional iodine affected all precursor preparation, radiofluorination, and deprotection steps. First, among the various methods tested (19-21), the reaction developed by Qin et al. (22) was solely amenable to producing a single iodine-substituted precursor 6 , because the ${ }^{18} \mathrm{~F}$-fluoride labile leaving groups are introduced via a substitution reaction with iodine. Second, 5 equivalents of $\mathrm{Cu}(\mathrm{OTf})_{2}$ were required to produce radiofluorinated intermediate compound 7, presumably because of the presence of multiple nitrogen atoms in the guanidine group and the additional iodine, both of which are also able to interact with copper. Finally, in the next deprotection step, a moderate temperature $\left(70^{\circ} \mathrm{C}\right)$ turned out to be effective in maintaining the recovery yield of 8 better than the initial harsh conditions by preventing temperaturedependent deiodination (8). The following HPLC regioisomer separation finally produced isomerically pure ${ }^{18} \mathrm{~F}-\mathrm{FIBG}(\mathbf{8 a})$ and ${ }^{18} \mathrm{~F}$-IFBG $(\mathbf{8 b})$.

The subsequent in vitro and in vivo studies revealed the tumor imaging potential of the isolated ${ }^{18}$ F-FIBG. At $1 \mathrm{~h}$ after injection, ${ }^{18}$ F-FIBG showed a biodistribution profile similar to that of ${ }^{125} \mathrm{I}-$ MIBG, enabling clear visualization of the tumor in the PET

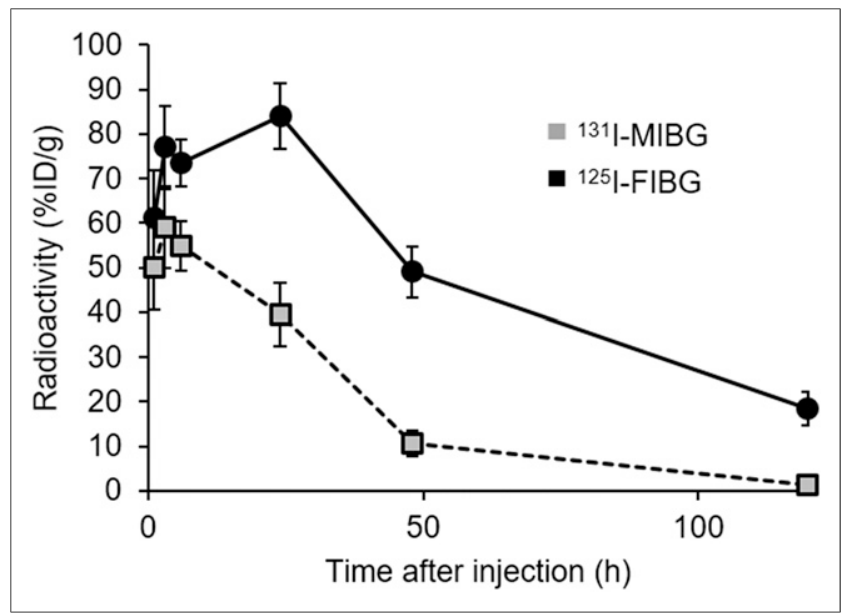

FIGURE 4. Tumor uptake of ${ }^{125}$ I-FIBG and ${ }^{131}$ I-MIBG in PC-12-bearing mice (mean $\pm \mathrm{SD}$, 3-5 per group). 


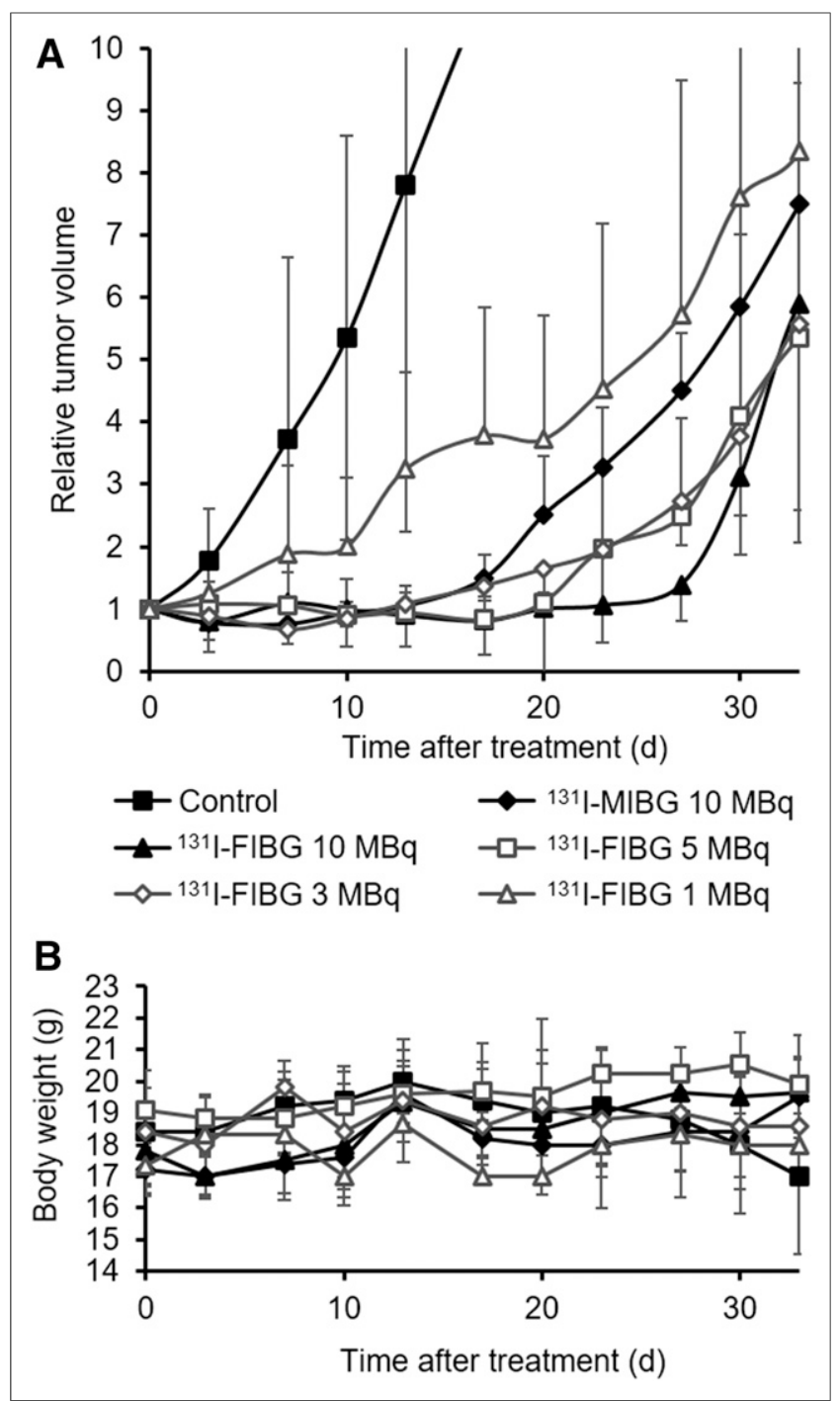

FIGURE 5. (A) Effect of ${ }^{131}$ I-FIBG and ${ }^{131}$ I-MIBG on growth of PC-12 tumor-bearing mice. (B) Body weight changes. Data are mean \pm SD (at least 5 per group, except 3 in ${ }^{131}$ I-FIBG 1 -MBq group).

images. Considering the clinical situation, a 24-h uptake period is required for single-photon imaging of ${ }^{123} \mathrm{I}-\mathrm{MIBG}$, because of the slow clearance of ${ }^{123} \mathrm{I}-\mathrm{MIBG}$ and the resolution issue. The similarly high initial background uptake of ${ }^{18} \mathrm{~F}$-FIBG may also be problematic. However, because our 2-h PET image showed an improved tumor-to-background ratio due to clearance from nontarget organs and persistent uptake within the tumor, the late-phase image may improve the detectability of ${ }^{18}$ F-FIBG. In addition, the advantage of PET probes over single-photon probes in terms of detectability has been well documented elsewhere (9). The first-inhuman trial of meta- ${ }^{18} \mathrm{~F}$-fluorobenzylguanidine indeed demonstrated that it can depict the tumor as early as $1 \mathrm{~h}$ after injection, despite its decreased tumor uptake versus *I-MIBG (1). In further studies, the tumor-imaging capability of ${ }^{18} \mathrm{~F}-\mathrm{FIBG}$ will be worth investigating in more clinically relevant models with various NET expression levels.

To evaluate whether we could use the regioisomer mixture without needing cumbersome HPLC separation, we also compared the properties of ${ }^{18} \mathrm{~F}-\mathrm{IFBG}$. Both in vitro and in vivo studies showed its reduced uptake in the PC-12 cells, though ${ }^{18} \mathrm{~F}-\mathrm{IFBG}$ and the regioisomer mixture also clearly depicted the $\mathrm{PC}-12$ tumor in the PET images. The distribution patterns of ${ }^{18} \mathrm{~F}-\mathrm{FIBG}$ and ${ }^{18} \mathrm{~F}-$ IFBG were comparable to their radioiodine-labeled counterparts, indicating that their radioactivity represents biodistribution of the intact compounds. The NET-positive cells take up MIBG derivatives by either NET-mediated specific uptake or passive diffusion (23). Because all NET inhibitors reduced uptake of each probe to a similar extent, and FIBG and IFBG should have comparable lipophilicities, the difference in their uptake may be attributed to their affinity for NET. These results suggest that the positions of iodine and fluorine in the aromatic ring affect the affinity for NET, in addition to the lipophilicity of the compound (24), and thus the regioisomer separation is recommended.

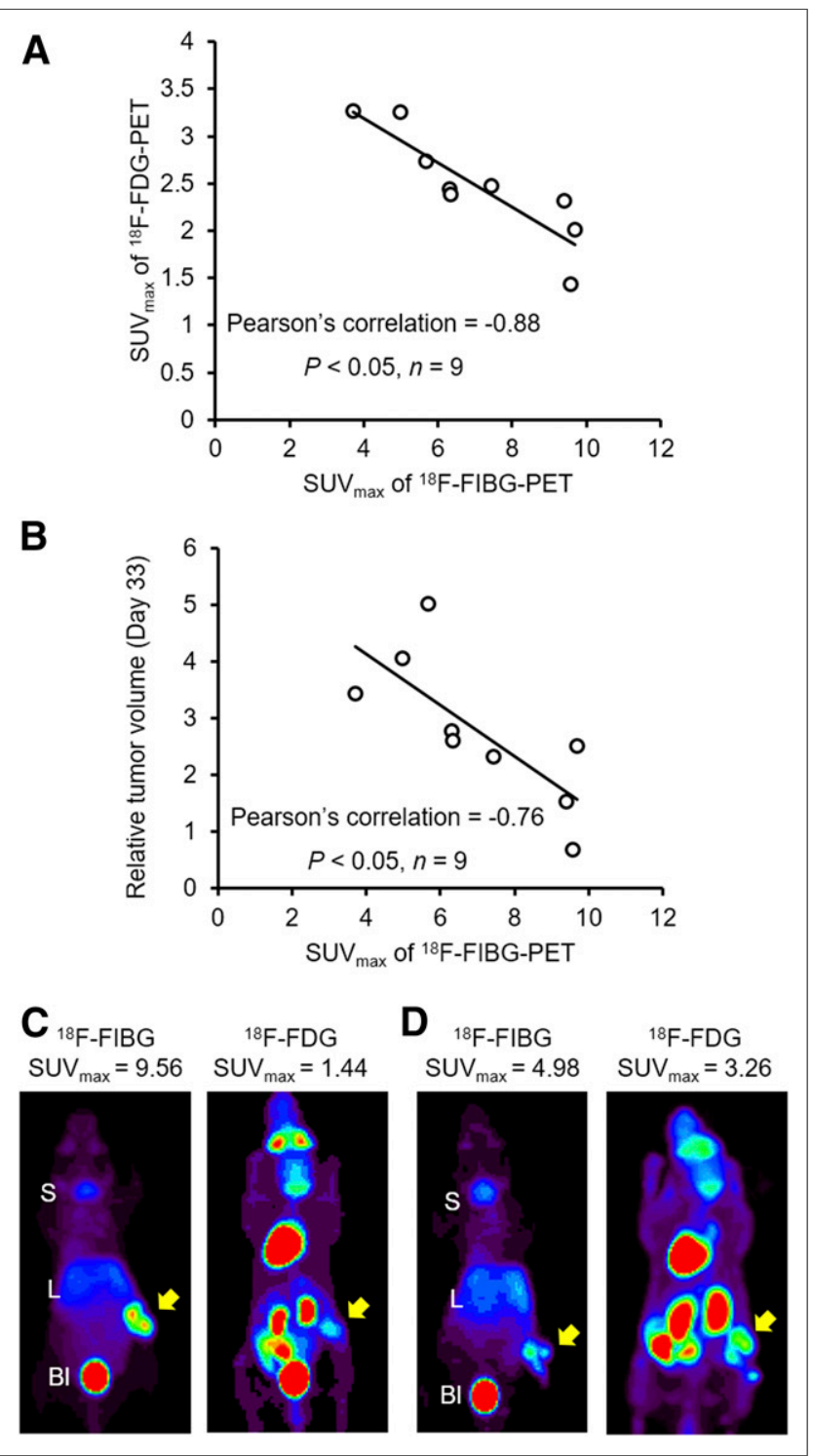

FIGURE 6. (A and B) Relationships between ${ }^{18} \mathrm{~F}-\mathrm{FIBG}$ uptake and ${ }^{18} \mathrm{~F}-$ FDG uptake (A) and between ${ }^{18} \mathrm{~F}-\mathrm{FIBG}$ uptake and relative tumor volume on day 33 after administration of $5 \mathrm{MBq}$ of ${ }^{131} \mathrm{I}-\mathrm{FIBG}$ (B). (C and D) Representative pretreatment ${ }^{18} \mathrm{~F}-\mathrm{FIBG}$ (left) and ${ }^{18} \mathrm{~F}-\mathrm{FDG}$ (right) PET images of mice with high (C) or low (D) ${ }^{18} \mathrm{~F}$-FIBG uptake. Arrows indicate tumor locations. $\mathrm{BI}=$ bladder; $\mathrm{L}=$ liver; $\mathrm{S}=$ salivary gland. 
The high and prolonged uptake of FIBG within the tumor shown in the long-term in vivo biodistribution studies suggests the possibility that ${ }^{131}$ I-FIBG improves therapeutic efficacy through an extended effective half-life in the tumor, along with the long physical half-life of ${ }^{131}$ I. Given that FIBG and MIBG showed comparable release profiles in vitro, various factors, such as reuptake in the surrounding cells, lipophilicity, and the deiodination tolerance of the compound, must have contributed differently in the biologic situation and caused prolonged retention of FIBG in the xenograft tumor.

As expected, ${ }^{131}$ I-FIBG demonstrated a better therapeutic effect than ${ }^{131}$ I-MIBG both in vitro and in vivo. The therapeutic efficacy of ${ }^{131}$ I-MIBG is limited because myelosuppression restricts the administration of an adequate tumoricidal dose to patients, though numerous clinical studies suggest a dose-dependent response rate for ${ }^{131} \mathrm{I}-\mathrm{MIBG}$ therapy (25). The estimated absorbed radiation dose in the red marrow was approximately 1.7 -fold higher for ${ }^{131} \mathrm{I}$-FIBG because it has a relatively higher normal-organ uptake than that of ${ }^{131}$ I-MIBG; therefore, the maximum tolerated dose of ${ }^{131} \mathrm{I}$-FIBG should be smaller than that of ${ }^{131}$ I-MIBG. However, because the ${ }^{131}$ I-FIBG 3-MBq group showed a $\mathrm{T}_{2.5}$ comparable to that of the ${ }^{131} \mathrm{I}$-MIBG $10-\mathrm{MBq}$ group, its high and prolonged tumor uptake would allow higher and more selective radiation dose exposure in the tumor. Further evaluation of the maximum tolerated dose and cumulative dose estimate in various tumor conditions is necessary to maximize the therapeutic efficacy of targeted radiation therapy with ${ }^{131} \mathrm{I}-\mathrm{FIBG}$ and consolidate its usefulness in comparison with ${ }^{131} \mathrm{I}-\mathrm{MIBG}$.

The correlation between the $\mathrm{SUV}_{\max }$ of ${ }^{18} \mathrm{~F}$-FIBG PET and the therapeutic effect of ${ }^{131}$ I-FIBG suggests the usefulness of pretherapy ${ }^{18} \mathrm{~F}$-FIBG PET for selecting patients suitable for ${ }^{131} \mathrm{I}$-FIBG therapy, in addition to its potential usefulness for detecting lesions and monitoring therapy. Radiation dose estimation methods for ${ }^{123}$ I-MIBG have not been established, primarily because of the semiquantitative nature and resolution issues, as well as the heterogeneous expression of NET at multiple sites (25). Although the applicability of a short-lived PET tracer such as ${ }^{18} \mathrm{~F}$ for estimating therapeutic absorbed doses requires further investigations, 2 retrospective studies exhibited an association between early lesion uptake and absorbed dose after PET scans with ${ }^{68}$ Ga-labeled somatostatin analogs $(26,27)$. To evaluate whether the pretherapy ${ }^{18} \mathrm{~F}-\mathrm{FIBG}$ uptake is associated with absorbed radiation dose in the tumor may be the objective of future research.

Of note, our data showed a strong inverse correlation between uptake levels of an MIBG analog and ${ }^{18}$ F-FDG, confirming several clinical studies that observed differential uptake patterns between MIBG and ${ }^{18} \mathrm{~F}-\mathrm{FDG}(3,28)$. This finding is likely due to the individual microenvironmental differences in the implanted area, such as accessibility to a large blood vessel. The poorly differentiated tumor sites are known to either decrease MIBG uptake in NETexpressing tumors or increase ${ }^{18} \mathrm{~F}$-FDG uptake in various types of cancer $(25,29,30)$. These results suggest that ${ }^{18} \mathrm{~F}$-FIBG, together with ${ }^{18} \mathrm{~F}-\mathrm{FDG}$, could be used to develop an optimum combination therapy for ${ }^{131}$ I-FIBG treatment (4) or therapeutic monitoring.

There were several limitations to our study. First, although we were able to simplify the radiolabeling method, the yield was still low and the procedures were rather complicated for clinical use, especially as regioisomer separation was required. Further studies are needed to optimize the radiosynthetic procedure, including selective synthesis of isomerically pure ${ }^{18} \mathrm{~F}$-FIBG and the development of an automated method. Second, the data need deliberate interpretation as we used only one xenograft model with exceptionally high NET expression levels, whereas clinical NET-expressing tumors express heterogeneous phenotypes. Nevertheless, the promising properties shown in this study suggest that FIBG warrants further evaluation in more clinically relevant models such as various neuroblastoma xenografts and their metastatic models.

\section{CONCLUSION}

We found that copper-mediated radiofluorination successfully produces ${ }^{18} \mathrm{~F}$-FIBG in 2 steps plus HPLC purification. Excellent tumor detectability and uptake comparable to MIBG were proven with ${ }^{18}$ F-FIBG. Moreover, ${ }^{131}$ I-FIBG showed a greater therapeutic effect in malignant pheochromocytomas than did ${ }^{131}$ I-MIBG. These results support the potential usefulness of FIBG as a diagnostic and therapeutic agent for the management of NET-expressing tumors.

\section{DISCLOSURE}

This work was supported by JSPS KAKENHI grant 26860981 to Aiko Yamaguchi. No other potential conflict of interest relevant to this article was reported.

\section{ACKNOWLEDGMENTS}

We thank Naoko Ichiishi (Department of Chemistry, University of Michigan) and Peter J. H. Scott (Department of Radiology and Interdepartmental Program in Medicinal Chemistry, University of Michigan) for providing expert technical advice on ${ }^{18} \mathrm{~F}$-labeling, and we thank Takashi Ogasawara (Cyclotron Facility, Gunma University Hospital) for producing ${ }^{18} \mathrm{~F}$-fluoride and ${ }^{18} \mathrm{~F}-\mathrm{FDG}$.

\section{REFERENCES}

1. Pandit-Taskar N, Modak S. Norepinephrine transporter as a target for imaging and therapy. J Nucl Med. 2017;58(suppl):39S-53S.

2. Kayano D, Kinuya S. Iodine-131 metaiodobenzylguanidine therapy for neuroblastoma: reports so far and future perspective. ScientificWorldJournal. 2015;2015:189135.

3. Sharp SE, Shulkin BL, Gelfand MJ, Salisbury S, Furman WL. ${ }^{123}$ I-MIBG scintigraphy and ${ }^{18}$ F-FDG PET in neuroblastoma. J Nucl Med. 2009;50:1237-1243.

4. Matthay KK, George RE, Yu AL. Promising therapeutic targets in neuroblastoma. Clin Cancer Res. 2012;18:2740-2753.

5. Vaidyanathan G, McDougald D, Koumarianou E, Choi J, Hens M, Zalutsky MR. Synthesis and evaluation of $4-\left[{ }^{18} \mathrm{~F}\right]$ fluoropropoxy-3-iodobenzylguanidine $\left(\left[{ }^{18} \mathrm{~F}\right]\right.$ FPOIBG): a novel ${ }^{18}$ F-labeled analogue of MIBG. Nucl Med Biol. 2015;42:673-684.

6. Zhang H, Huang R, Cheung NK, et al. Imaging the norepinephrine transporter in neuroblastoma: a comparison of $\left[{ }^{18} \mathrm{~F}\right]-\mathrm{MFBG}$ and ${ }^{123} \mathrm{I}-\mathrm{MIBG}$. Clin Cancer Res. 2014;20:2182-2191.

7. Gaertner FC, Wiedemann T, Yousefi BH, et al. Preclinical evaluation of ${ }^{18} \mathrm{~F}-$ LMI1195 for in vivo imaging of pheochromocytoma in the MENX tumor model. J Nucl Med. 2013;54:2111-2117.

8. Vaidyanathan G, Affleck DJ, Zalutsky MR. (4-[ $\left.{ }^{18} \mathrm{~F}\right]$ fluoro-3-iodobenzyl)guanidine, a potential MIBG analogue for positron emission tomography. J Med Chem. 1994;37:3655-3662.

9. Baum RP, Kulkarni HR, Carreras C. Peptides and receptors in image-guided therapy: theranostics for neuroendocrine neoplasms. Semin Nucl Med. 2012;42: 190-207.

10. Vaidyanathan G, Affleck DJ, Zalutsky MR. Validation of 4-[fluorine-18]fluoro-3iodobenzylguanidine as a positron-emitting analog of MIBG. J Nucl Med. 1995; 36:644-650.

11. Vaidyanathan G, Zhao XG, Strickland DK, Zalutsky MR. No-carrier-added iodine-131-FIBG: evaluation of an MIBG analog. J Nucl Med. 1997;38:330-334.

12. Preshlock S, Tredwell M, Gouverneur V. ${ }^{18} \mathrm{~F}$-Labeling of arenes and heteroarenes for applications in positron emission tomography. Chem Rev. 2016;116:719-766.

13. Rotstein BH, Wang L, Liu RY, et al. Mechanistic studies and radiofluorination of structurally diverse pharmaceuticals with spirocyclic iodonium(III) ylides. Chem Sci. 2016;7:4407-4417.

14. Preshlock S, Calderwood S, Verhoog S, et al. Enhanced copper-mediated ${ }^{18} \mathrm{~F}$ fluorination of aryl boronic esters provides eight radiotracers for PET applications. Chem Commun (Camb). 2016;52:8361-8364. 
15. Hu B. Vävere AL, Neumann KD, Shulkin BL, DiMagno SG, Snyder SE. A practical, automated synthesis of meta- $\left[{ }^{18} \mathrm{~F}\right]$ fluorobenzylguanidine for clinical use. ACS Chem Neurosci. 2015;6:1870-1879.

16. Ichiishi N, Brooks AF, Topczewski JJ, Rodnick ME, Sanford MS, Scott PJH. Copper-catalyzed $\left[{ }^{18} \mathrm{~F}\right]$ fluorination of (mesityl)(aryl)iodonium salts. Org Lett. 2014;16:3224-3227.

17. Vaidyanathan G, Affleck DJ, Alston KL, Zalutsky MR. A tin precursor for the synthesis of no-carrier-added [*I]MIBG and [ $\left.{ }^{211} \mathrm{At}\right] \mathrm{MABG}$. J Labelled Comp Radiopharm. 2007;50:177-182.

18. Watanabe S, Hanaoka H, Liang JX, Iida Y, Endo K, Ishioka NS. PET imaging of norepinephrine transporter-expressing tumors using ${ }^{76} \mathrm{Br}$-meta-bromobenzylguanidine. J Nucl Med. 2010;51:1472-1479.

19. Mossine AV, Brooks AF, Makaravage KJ, et al. Synthesis of $\left[{ }^{18} \mathrm{~F}\right]$ arenes via the copper-mediated $\left[{ }^{18}\right.$ F]fluorination of boronic acids. Org Lett. 2015;17:57805783.

20. Tredwell M, Preshlock SM, Taylor NJ, et al. A general copper-mediated nucleophilic ${ }^{18} \mathrm{~F}$ fluorination of arenes. Angew Chem Int Ed Engl. 2014;53:77517755 .

21. Qiu D, Mo F, Zheng Z, Zhang Y, Wang J. Gold(III)-catalyzed halogenation of aromatic boronates with N-halosuccinimides. Org Lett. 2010;12:5474-5477.

22. Qin L, Hu B, Neumann KD, et al. A mild and general one-pot synthesis of densely functionalized diaryliodonium salts. European J Org Chem. 2015;2015: 5919-5924.
23. Jaques S Jr, Tobes MC, Sisson JC. Sodium dependency of uptake of norepinephrine and m-iodobenzylguanidine into cultured human pheochromocytoma cells: evidence for uptake-one. Cancer Res. 1987;47:3920-3928.

24. Vaidyanathan G, Shankar S, Affleck DJ, Welsh PC, Slade SK, Zalutsky MR. Biological evaluation of ring- and side-chain-substituted m-iodobenzylguanidine analogues. Bioconjug Chem. 2001;12:798-806.

25. Grünwald F, Ezziddin S. ${ }^{131}$ I-metaiodobenzylguanidine therapy of neuroblastoma and other neuroendocrine tumors. Semin Nucl Med. 2010;40:153-163.

26. Hänscheid H, Sweeney RA, Flentje M, et al. PET SUV correlates with radionuclide uptake in peptide receptor therapy in meningioma. Eur J Nucl Med Mol Imaging. 2012;39:1284-1288.

27. Ezziddin S, Lohmar J, Yong-Hing CJ, et al. Does the pretherapeutic tumor SUV in ${ }^{68} \mathrm{Ga}$ DOTATOC PET predict the absorbed dose of ${ }^{177} \mathrm{Lu}$ octreotate? Clin Nucl Med. 2012;37:e141-e147.

28. Melzer HI, Coppenrath E, Schmid I, et al. ${ }^{123}$ I-MIBG scintigraphy/SPECT versus ${ }^{18}$ F-FDG PET in paediatric neuroblastoma. Eur J Nucl Med Mol Imaging. 2011;38:1648-1658.

29. Vesselle H, Salskov A, Turcotte E, et al. Relationship between non-small cell lung cancer FDG uptake at PET, tumor histology, and Ki-67 proliferation index. J Thorac Oncol. 2008;3:971-978.

30. Seo S, Hatano E, Higashi T, et al. Fluorine-18 fluorodeoxyglucose positron emission tomography predicts tumor differentiation, P-glycoprotein expression, and outcome after resection in hepatocellular carcinoma. Clin Cancer Res. 2007;13:427-433. 